\title{
Complicaciones asociadas al catéter percutáneo en recién nacidos pretérmino y a término
}

\author{
Martha Alicia Higareda-Almaraz', Paz Alejandra Gutiérrez-Monraz², Ruth Alejandrina Castillo-Sánchez², \\ Juan Carlos Barrera-de León ${ }^{3}$, Ana Bertha Zavalza-Gómez² y Enrique Higareda-Almaraz ${ }^{1}$ \\ ${ }^{1}$ Jefatura de Servicios de Prestaciones Médicas, Instituto Mexicano del Seguro Social, Colima, Col.; ${ }^{2}$ UMAE, Hospital de Gineco-Obstetricia; ${ }^{3}$ UMAE, \\ Hospital de Pediatría. Centro Médico Nacional de Occidente, Instituto del Mexicano Seguro Social, Guadalajara, Jal., México
}

\section{Resumen}

Objetivo: Identificar las complicaciones asociadas al catéter percutáneo en recién nacidos pretérmino y a término. Método: Estudio transversal comparativo. Se incluyeron recién nacidos que tenían insertado un catéter percutáneo, con resultados de cultivo de sangre y segmento distal del catéter. Se formaron dos grupos: pretérmino y a término. Se calcularon la razón de momios (RM) y el intervalo de confianza del 95\% (IC 95\%). Resultados: Se analizaron datos de 50 recién nacidos por grupo. En los pretérmino y a término se encontró un valor de la media de edad gestacional de $30 \pm 3$ y $40 \pm 2$, respectivamente $(p=0.01)$, y unos porcentajes de sepsis por catéter del 36 y el 18\% ( $p=0.02 ; R M: 2.56$; IC 95\%: 1.02-7.17), de catéter infectado del 50 y el 22\% ( $p=0.01$; RM: 5.92; IC 95\%: 1.66-23.12), de catéter colonizado del 24 y el 14\% ( $p=0.01$; RM: 3.58; IC 95\%: 1.32-9.90), de infección local del 14 y el 8\% ( $p=0.03 ; R M: 1.87 ;$ IC 95\%: 1.45-8.29), de infiltración del 18 y el 4\% ( $p=0.02$; RM: 5.27; IC 95\%: 1.17-59), de retiro accidental del 6 y el 22\% ( $p=0.02 ; R M: 0.23 ;$ IC 95\%: $0.05-0.87)$ y de rotura del catéter del 10 y el 28\% ( $p=0.02 ; R M: 0.29 ;$ IC 95\%: 0.08-0.98). Conclusiones: Se encontró mayor asociación de infecciones e infiltraciones por catéter percutáneo en los pretérmino, y de retiro accidental y rotura del catéter en los nacidos a término.

PALABRAS CLAVE: Catéter percutáneo. Complicaciones. Recién nacidos.

\begin{abstract}
Objective: Identify percutaneous catheter-related complications in preterm and term newborns. Methods: Comparative cross-section. Were included newborns whit percutaneous catheter insertion, blood culture results and distal catheter segment. Were formed two groups: Preterm and term. Results: Were analyzed the data of preterm $(n=50)$ and term $(n=50)$ newborn, the gestational age was $30 \pm 3$ and $40 \pm 2(p=0.01)$. The frecuency in preterm and term newborn was respectively, sepsis catheter 36 and 18\% ( $p=0.02$; OR: 2.56; 95\% Cl: 1.02-7.17), infected catheter 50 and 22\% ( $p=0.01 ;$ OR: 5.92; 95\% Cl: 1.66-23.12), colonized catheter of 24 and 14\% ( $p=0.01$; OR: 3.58; 95\% Cl: 1.32-9.90), local infection 14 and 8\% ( $p=0.03$; OR: $1.87 ; 95 \% \mathrm{Cl}: 1.45-8.29)$, infiltration 18 and $4 \%(p=0.02$; OR: 5.27; 95\% Cl: 1.17-59), accidental removal 6 and $22 \%$ ( $p=0.02$; OR: 0.23; 95\% Cl: 0.05-0.87) and catheter rupture 10 and 28\% ( $p=0.02 ;$ OR: 0.29; 95\% Cl: 0.08-0.98). Conclusions: We found a higher association of infections and infiltrations by percutaneous catheter in preterm and term prevailed in accidental removal and catheter rupture.
\end{abstract}

KEY WORDS: Percutáneous catheter. Complications. Newborn.

\section{Correspondencia:}

Martha Alicia Higareda-Almaraz

Prisciliano Sánchez, 530

C.P. 44100 Guadalajara, Jal., México

E-mail: marthahigareda12@ hotmail.com
Fecha de recepción en versión modificada: 27-07-2016

Fecha de aceptación: 08-08-2016

DOI: //dx.doi.org/10.24875/GMM.17002791
Gac Med Mex. 2018;154:47-53

Contents available at PubMed www.gacetamedicademexico.com 


\section{Introducción}

Es importante tener un acceso venoso seguro en los recién nacidos hospitalizados; esto se logra con la inserción de un catéter percutáneo, el cual se desplaza en una vena periférica y se coloca en la vena cava superior ${ }^{1}$.

Los catéteres percutáneos fueron utilizados inicialmente por Shaw en niños de bajo peso. Esta técnica se utilizó para nutrición parenteral y demostró ser segura, evita dolor, múltiples punciones e inmovilizaciones $^{2}$. Actualmente, los catéteres percutáneos de Silastic $₫$ son los más utilizados por su fácil colocación y menor número de complicaciones. Sin embargo, se han reportado eventos adversos ${ }^{3}$. Algunos autores han descrito que la prematuridad y el uso de nutrición parenteral son factores de riesgo para sepsis por catéter, la cual se ha reportado en el $23 \%$ de los catéteres ${ }^{4,5}$.

Algunas complicaciones están asociadas con la mala posición inicial del catéter o un desplazamiento dentro de la vena, y en algunas ocasiones se perfora la vena con extravasación de líquidos. Estos factores pueden ocasionar infiltraciones (5\%), retiro accidental $(2 \%)$, derrame pleural (1\%), pericarditis y taponamiento cardiaco $(1 \%)^{6-8}$.

La baja tolerancia a las infusiones con presión a través del catéter percutáneo puede ocasionar la rotura del catéter $(9 \%)^{6,9}$.

La presencia de trombos de sangre, fibrina o minerales precipitados en el catéter produce oclusión del catéter, que se reporta en un $19 \% \%^{6,10}$.

Actualmente se desconoce en qué edad gestacional son más frecuentes los eventos adversos por inserción del catéter percutáneo. Por lo tanto, el objetivo de esta investigación fue identificar las complicaciones asociadas al catéter percutáneo en los recién nacidos pretérmino y a término.

\section{Métodos}

El estudio fue realizado de enero a diciembre de 2015 en el servicio de neonatología de la Unidad Médica de Alta Especialidad del Hospital de Gineco-Obstetricia del Instituto Mexicano del Seguro Social en Guadalajara, México.

Fueron incluidos los expedientes clínicos de los recién nacidos hospitalizados de cualquier sexo y peso que requirieron inserción de catéter percutáneo para nutrición parenteral, con resultado de hemocultivo y cultivo del segmento distal del catéter percutáneo. Fueron excluidos los recién nacidos con expedientes clínicos incompletos y pérdida de los resultados de cultivos en sangre y del catéter percutáneo.

Se utilizó la fórmula para dos proporciones para determinar el tamaño de muestra. El porcentaje esperado de complicaciones en los pretérmino es del $65 \%$ y en los a término es del $40 \%$. Se calculó con un poder del $80 \%$ y se obtuvo un tamaño de muestra estimado de 49.

Fueron revisados 100 expedientes clínicos de recién nacidos con inserción de catéter percutáneo, marca Vygon, de 2 Fr. Un investigador recolectó los datos, y la información obtenida se registró en un cuestionario estructurado previamente realizado.

Edad gestacional valorada por la nueva escala de Ballard ${ }^{11}$, peso y sexo al nacimiento, número de días que permaneció insertado el catéter percutáneo, y resultado de cultivo de sangre y del segmento distal del catéter percutáneo fueron recabados. También se registraron datos clínicos (temperatura axilar en grados centígrados, frecuencia cardiaca en latidos por minuto, presión arterial en $\mathrm{mmHg}$, cambios de coloración, llenado capilar en segundos, incremento en soporte ventilatorio y apneas) y de laboratorio (número de leucocitos, plaquetas y neutrófilos).

\section{Definición de las complicaciones investigadas}

- Catéter colonizado: aislamiento de microorganismos en el segmento distal del catéter percutáneo por cultivo cuantitativo con un número $<100,000$ unidades formadoras de colonias (UFC).

- Catéter infectado: aislamiento de microorganismos en el segmento distal del catéter percutáneo por cultivo cuantitativo con un número $\geq 100,000$ UFC.

- Infección local: presentar dos o más signos y síntomas en el punto de inserción del catéter percutáneo (dolor, eritema, inflamación, exudado purulento o cordón venoso palpable).

- Sepsis por catéter percutáneo: aislamiento de microorganismos en el segmento distal del catéter percutáneo en número $\geq 100,000$ UFC en cultivo cuantitativo y hemocultivo positivo para el mismo microorganismo, acompañado de la presencia de síntomas de acuerdo con los criterios del consenso para sepsis en neonatos ${ }^{12}$. Datos clínicos: inestabilidad en la curva térmica (temperatura 
axilar $<36.50>37.5^{\circ} \mathrm{C}$ ), taquicardia (frecuencia cardiaca $>180$ latidos por minuto), hipoperfusión, incremento en soporte ventilatorio, apneas. Datos de laboratorio: leucocitos $>20,000$ células $/ \mathrm{mm}^{3}$ o leucocitopenia $<5,000$ células $/ \mathrm{mm}^{3}$, trombocitopenia $<100,000$ células $/ \mathrm{mm}^{3}$, neutropenia $<1,500$, relación banda / neutrófilos $>0.2$, y elevación de proteína $C$ reactiva $>5 \mathrm{mg} / \mathrm{l}$.

- Probable sepsis por catéter: presencia de datos clínicos para sepsis con hemocultivo y cultivo de segmento distal del catéter percutáneo negativos. El cuadro clínico remite dentro de las 48 horas siguientes a la retirada del catéter.

- Rotura del catéter en la zona de inserción: presencia de salida de líquido en el sitio de fijación.

- Retiro accidental: desplazamiento del sitio de colocación con salida del catéter percutáneo.

- Infiltración: acumulación de sustancia infundida en el tejido que circunda la vena, debido al desplazamiento del catéter de la íntima de la vena hacia el tejido subcutáneo.

- Derrame pleural: acumulación patológica de líquido en el espacio pleural.

- Derrame pericárdico: acumulación anormal de líquido en la cavidad pericárdica, que eleva la presión intrapleural y de este modo ejerce un efecto negativo sobre la función del corazón, ocasionando un taponamiento cardiaco.

- Oclusión: incapacidad para infundir líquido, confirmada por una enfermera y un médico.

- Defunción.

\section{Técnicas de cultivo}

- Hemocultivo: las muestras de sangre para hemocultivo se tomaron según la técnica convencional ( $1 \mathrm{ml}$ de sangre periférica inoculada en un tubo pediátrico con medio para cultivo Bact / Alert $₫$ PF pediatric). Posteriormente fueron monitorizadas y analizadas en un sistema automatizado BacT / Alert 120, por 7 días. El resultado del hemocultivo positivo fue examinado en microscopio y subcultivado en agar chocolate, para anaerobios y agar Sabouraud, acorde con el método estándar. La evaluación para determinar el crecimiento en estos cultivos se realizó en los días 3, 7 y 14 tras la siembra. Para establecer un diagnostico definitivo de sepsis por hongos y bacterias fue necesario el aislamiento de estos en los cultivos. Este crecimiento se reportó como positivo o negativo, acordando la manufactura de las recomendaciones del MicroScan $® 96$. El tiempo hasta obtener el resultado se consideró en un rango de 2 días a 2 semanas. La duración más larga fue para considerar cultivo negativo para hongos o bacterias.

- Cultivo cuantitativo de catéter percutáneo: se pasó $1 \mathrm{ml}$ de agua destilada estéril por el lumen del catéter percutáneo y se sometió a vórtex durante 1 minuto. Luego se sembró $0,1 \mathrm{ml}$ de esta suspensión en placa de agar y se incubó durante 7 días. Se consideró cultivo positivo la presencia de microorganismos.

Para comparar la diferencia de proporciones entre los grupos se utilizó la prueba de ji al cuadrado, y para la diferencia de medias se utilizó la t de Student. Para identificar la asociación de complicaciones por catéter percutáneo con la edad gestacional se utilizó la razón de momios (RM) con sus intervalos de confianza del 95\% (IC95\%). Para un análisis ajustado se realizó regresión logística. Se consideró significación estadística un valor de $p<0.05$. Se utilizó el programa SPSS (Chicago IL versión 19.0) para realizar el análisis estadístico.

Este estudio siguió los principios éticos de la investigación científica contenidos en la declaración de Helsinki ${ }^{13}$. El protocolo no requirió consentimiento informado y fue aceptado por el comité de investigación (número de registro: R-2012-1310-37).

\section{Resultados}

Se revisaron 1020 expedientes clínicos de recién nacidos. De estos, 920 (90\%) tuvieron inserción de catéter percutáneo, de los cuales fueron excluidos 820 . Se incluyeron 100 recién nacidos (50 pretérmino y 50 a término), en los cuales se investigaron las complicaciones por inserción de catéter percutáneo (Fig. 1).

En la tabla 1 se muestran las características clínicas de los recién nacidos. Se encontraron diferencias estadísticamente significativas en los recién nacidos pretérmino/término, en peso $(1404 \pm 59 / 2922 \pm 68 ; p=0.01)$, edad gestacional $(30 \pm 2 / 40 \pm 2 ; p=0.01)$, resultado de hemocultivo positivo (17 [34\%] / 9 [18\%]; $p=0.05$ ) y cultivo del segmento distal del catéter percutáneo (37 [74\%] / 18 [36\%]; $p=0.01$ ).

En la tabla 2 se muestran las complicaciones por catéter percutáneo en los recién nacidos pretérmino / termino. Se encontró sepsis por catéter en 18 (36\%) / 9 (18\%) (RM: 2.56; $p=0.02$ ), catéter colonizado en 12 (24\%) / 7 (14\%) (RM: 3.58; $p=0.01)$, catéter infectado 


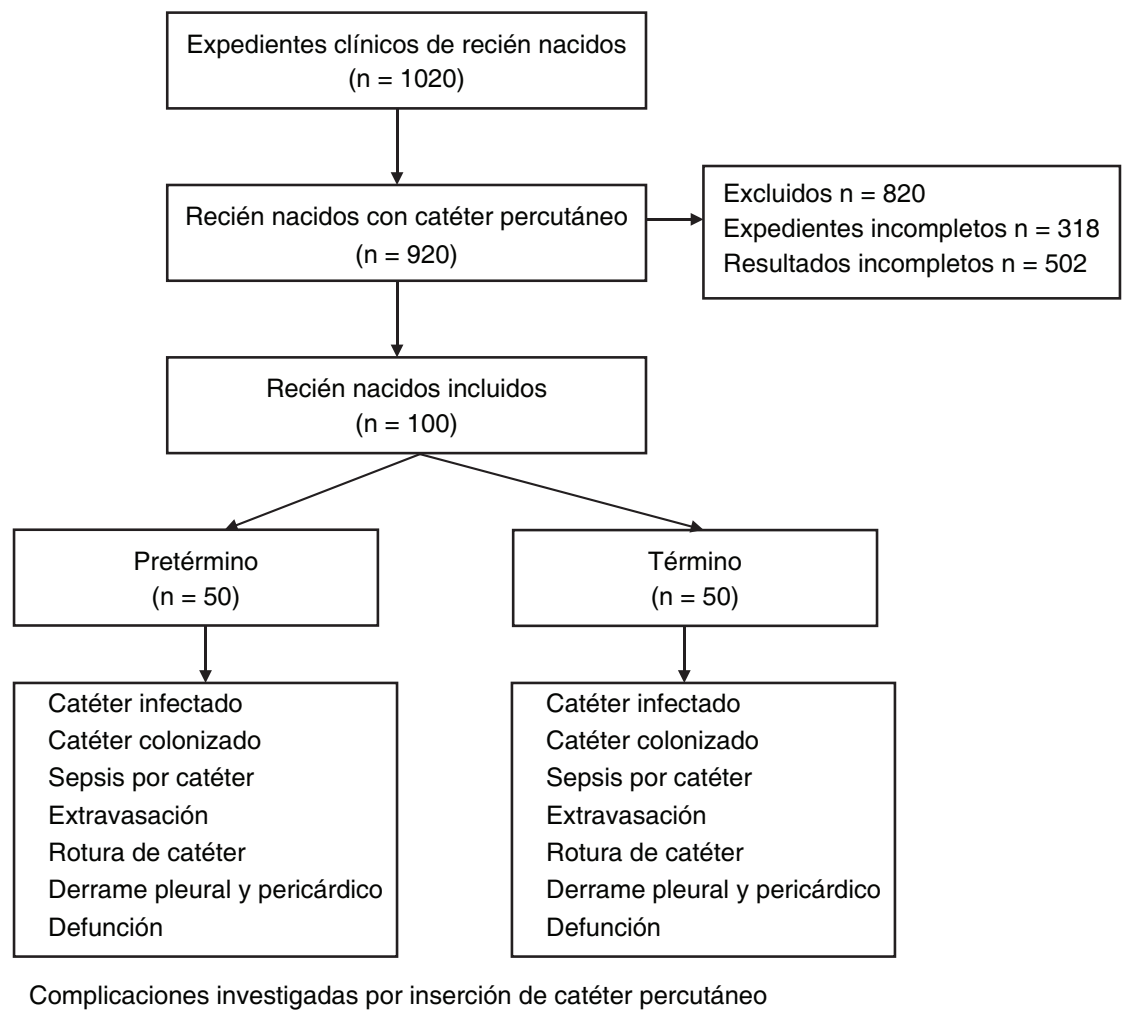

Figura 1. Perfil del estudio.

Tabla 1. Comparación de las características clínicas en los recién nacidos pretérmino y a término con inserción de catéter percutáneo

\begin{tabular}{lccc}
\hline Características clínicas & \multicolumn{2}{c}{ Grupos } & p \\
\cline { 2 - 3 } & $\begin{array}{l}\text { Pretérmino } \\
\mathbf{n = 5 0}(\%)\end{array}$ & $\begin{array}{c}\text { Término } \\
\mathbf{n}=\mathbf{5 0}(\%)\end{array}$ \\
\hline Peso nacimiento, media \pm DE & $1404 \pm 59$ & $2922 \pm 68$ & 0.01 \\
Edad gestacional, media \pm DE & $30 \pm 2$ & $40 \pm 2$ & 0.01 \\
Sexo masculino, $n$ (\%) & $23(46)$ & $22(44)$ & 0.50 \\
$\begin{array}{lccc}\text { Edad de inserción del catéter, } \\
\text { media } \pm \text { DE }\end{array}$ & $8 \pm 2$ & $7 \pm 2$ & 0.27 \\
$\begin{array}{l}\text { Días con catéter percutáneo, } \\
\text { media } \pm \text { DE }\end{array}$ & $10 \pm 3$ & $12 \pm 2$ & 0.35 \\
$\begin{array}{l}\text { Uso de nutrición parenteral, (\%) } \\
\text { Hemocultivo positivo, } n \text { (\%) }\end{array}$ & $50(100)$ & $50(100)$ & 1.00 \\
$\begin{array}{l}\text { Cultivo de catéter percutáneo } \\
\text { positivo, } n \text { (\%) }\end{array}$ & $37(74)$ & $18(36)$ & 0.01 \\
\hline
\end{tabular}

Para comparar la diferencia de medias entre los grupos se utilizó la prueba $t$ de Student. Para comparar la diferencia de proporciones entre los grupos se utilizó la prueba de ji al cuadrado.

DE: desviación estándar

en 25 (50\%) / 11 (22\%) (RM: 5.92; $p=0.01)$, infección local en $6(12 \%)$ / 4 (8\%) (RM: 1.87; $p=0.03)$, infiltración en 9 (18) / 2 (4) (RM: 5.27; $p=0.02)$, rotura de catéter en $5(10 \%) / 14(28 \%)(R M: 0.29 ; p=0.02)$, retiro accidental en $3(6 \%) / 11(22 \%)$ (RM: 0.23; $p=0.02)$, infiltración en 9 (18\%) / 2 (4\%) (RM: 5.27; $p=0.02)$. En otras variables no se encontró significación estadística.

Al calcular una regresión logística, introduciendo en la ecuación las variables en términos categóricos, se encontró que permanecieron asociadas las complicaciones con la edad gestacional en los recién nacidos pretérmino de manera significativa con las variables sepsis por catéter $(p=0.03)$, catéter infectado $(p=0.04)$, infiltración $(p=0.02)$ y probable sepsis por catéter $(p=0.01)$ (Tabla 3$)$.

En los resultados de los hemocultivos y cultivos del segmento distal del catéter percutáneo se encontraron bacterias y hongos. En el hemocultivo se encontró con mayor frecuencia Staphylococcus epidermidis en el grupo de los pretérmino (11 [24\%]) que en el grupo de los a término (4 [8\%]; $p=0.01$ ). En los resultados de los cultivos del catéter percutáneo se encontró un mayor porcentaje de $S$. epidermidis, respectivamente en los pretérmino / termino, $18(36 \%) / 8(16 \%)(p=0.01)$ y de Staphylococcus aureus, $8(16 \%) / 2(4 \%)(p=0.01)$ (Tabla 4).

La vena en la que se insertó con mayor frecuencia el catéter percutáneo fue la axilar derecha, en 11 
Tabla 2. Complicaciones asociadas al catéter percutáneo en los recién nacidos pretérmino y a término

\begin{tabular}{|c|c|c|c|c|c|}
\hline \multirow[t]{2}{*}{ Complicaciones } & \multicolumn{2}{|c|}{ Grupos } & \multirow[b]{2}{*}{ RM } & \multirow[b]{2}{*}{ IC 95\% } & \multirow[b]{2}{*}{$p$} \\
\hline & $\begin{array}{l}\text { Pretérmino } \\
n=50\end{array}$ & $\begin{array}{c}\text { Término } \\
n=50\end{array}$ & & & \\
\hline Catéter colonizado, n (\%) & $12(24)$ & $7(14)$ & 3.58 & $1.32-9.90$ & 0.01 \\
\hline Catéter infectado, n (\%) & $25(50)$ & $11(22)$ & 5.92 & $1.66-23.12$ & 0.01 \\
\hline Infección local, n (\%) & $7(14)$ & $4(8)$ & 1.87 & $1.45-8.29$ & 0.03 \\
\hline Sepsis por catéter, n (\%) & $18(36)$ & $9(18)$ & 2.56 & $1.02-7.17$ & 0.02 \\
\hline Probable sepsis por catéter, $\mathrm{n}(\%)$ & $8(16)$ & $2(4)$ & 4.57 & $1.00-6.25$ & 0.04 \\
\hline Rotura de catéter en inserción, n (\%) & $5(10)$ & $14(28)$ & 0.29 & $0.08-0.98$ & 0.02 \\
\hline Retiro accidental, n (\%) & $3(6)$ & $11(22)$ & 0.23 & $0.05-0.97$ & 0.02 \\
\hline Infiltración, n (\%) & $9(18)$ & $2(4)$ & 5.27 & $1.17-59.00$ & 0.02 \\
\hline Derrame pericárdico, n (\%) & $1(2)$ & $1(2)$ & 1.00 & $0.00-37.84$ & 1.00 \\
\hline Derrame pleural, n (\%) & $1(2)$ & $1(2)$ & 1.00 & $0.00-37.84$ & 1.00 \\
\hline Oclusión, n (\%) & $7(14)$ & $6(12)$ & 1.19 & $0.33-4.43$ & 0.77 \\
\hline Defunción, n (\%) & $3(6)$ & $3(6)$ & 1.00 & $0.15-6.63$ & 1.00 \\
\hline
\end{tabular}

Para comprar la diferencia de proporciones entre los grupos se utilizó la prueba de ji al cuadrado.

IC 95\%: intervalo de confianza del 95\%; RM: razón de momios.

Tabla 3. Modelo logístico. Complicaciones asociadas al catéter percutáneo en los recién nacidos pretérmino y a término

\begin{tabular}{lccc}
\hline Complicaciones del catéter & $\mathbf{p}$ & $\mathbf{R M}$ & IC 95\% \\
\hline Infectado & 0.01 & 5.92 & $1.66-23.12$ \\
Sepsis por catéter & 0.01 & 3.91 & $1.00-29.00$ \\
Probable sepsis por catéter & 0.01 & 2.36 & $1.00-6.25$ \\
Infiltración & 0.02 & 5.27 & $1.37-59.00$ \\
\hline IC 95\% intervalo de confianza del 95\%: RM: razón de momios. &
\end{tabular}

recién nacidos, de los cuales fueron pretérmino 6 $(12 \%)$ y a término $5(10 \%)(p=0.74)$ (Tabla 5$)$.

\section{Discusión}

En el presente estudio se encontró una mayor asociación de infecciones e infiltraciones por catéter percutáneo en los recién nacidos pretérmino. En los recién nacidos a término hubo una mayor proporción de roturas y de retiro accidental.

Estos datos son importantes en el contexto clínico para realizar estrategias para tratar de corregir estas complicaciones y disminuir las secuelas, las cuales en algunas ocasiones pueden ser irreversibles.

Los catéteres percutáneos se utilizan frecuentemente en los recién nacidos hospitalizados para mantener un acceso venoso seguro'.

Un $80 \%$ de las infecciones sanguíneas se asocian a un catéter percutane $0^{14}$. Algunos autores han encontrado sepsis en el $23 \%$ de los $\operatorname{casos}^{5}$. Entre los factores de riesgo para presentar infección por la inserción de un catéter percutáneo se encuentran la prematuridad, las manipulaciones repetidas del catéter, el número de días de permanencia del catéter y la utilización para nutrición parentera ${ }^{15}$. Estos datos apoyan los resultados encontrados en esta investigación, en la que se halló un mayor porcentaje de sepsis en los recién nacidos pretérmino en comparación con los a término. Los patógenos pueden llegar al catéter a través de líquidos de infusión contaminados o por vía hematógena con las bacterias presentes en la piel, las cuales se encuentran en contacto con la entrada del catéter y a través de las conexiones ${ }^{16,17}$. Algunos autores han descrito que el estafilococo es la bacteria que se encuentra con mayor frecuencia en los cultivos de catéteres percutáneos ${ }^{18,19}$. En esta investigación encontramos resultados similares.

El desplazamiento del catéter es frecuente, ya que no tiene ningún dispositivo especial de fijación y se trata de pacientes muy difíciles de mantener inmóviles $^{20}$. Por lo tanto, es importante la visualización radiológica para determinar la posición del catéter ${ }^{21}$. Algunas publicaciones han demostrado complicaciones del catéter percutáneo por mala posición ${ }^{22,23} 0$ desplazamiento del catéter ${ }^{24}$. Entre estas se encuentra la salida accidental del catéter en un $2.3 \%$. En nuestra investigación encontramos mayor proporción en los recién nacidos a término que en los pretérmino, debido a que los recién 
Tabla 4. Comparación de los resultados de los cultivos en los recién nacidos pretérmino y a término con inserción de catéter percutáneo

\begin{tabular}{|c|c|c|c|}
\hline \multirow[t]{2}{*}{ Resultado de cultivos } & \multicolumn{2}{|c|}{ Grupos } & \multirow[t]{2}{*}{ p } \\
\hline & $\begin{array}{l}\text { Pretérmino } \\
n=50\end{array}$ & $\begin{array}{c}\text { Término } \\
n=50\end{array}$ & \\
\hline \multicolumn{4}{|l|}{ Hemocultivo } \\
\hline Candida albicans, n (\%) & $2(4)$ & $0(0)$ & 0.50 \\
\hline Staphylococcus epidermidis, n (\%) & $11(22)$ & $4(8)$ & 0.04 \\
\hline Staphylococcus haemolyticus, n (\%) & $2(4)$ & $0(0)$ & 0.50 \\
\hline Enterobacter cloacae, n (\%) & $1(2)$ & $0(0)$ & 0.50 \\
\hline Serratia marcescens, n (\%) & $1(2)$ & $0(0)$ & 0.50 \\
\hline Staphylococcus hominis, n (\%) & $0(0)$ & $1(2)$ & 0.50 \\
\hline Staphylococcus faecium, n (\%) & $0(0)$ & $1(2)$ & 0.50 \\
\hline Pseudomonas aeruginosa, $\mathrm{n}(\%)$ & $0(0)$ & $1(2)$ & 0.50 \\
\hline Klebsiella pneumoniae, n (\%) & $0(0)$ & $2(4)$ & 0.50 \\
\hline Negativo, n (\%) & $33(66)$ & $41(82)$ & 0.05 \\
\hline \multicolumn{4}{|l|}{ Catéter percutáneo } \\
\hline Staphylococcus epidermidis, n (\%) & $18(36)$ & $9(18)$ & 0.04 \\
\hline Enterobacter cloacae, n (\%) & $2(4)$ & $2(4)$ & 1.00 \\
\hline Pseudomonas aeruginosa, $\mathrm{n}(\%)$ & $0(0)$ & $1(2)$ & 0.50 \\
\hline Staphylococcus aureus, n (\%) & $8(16)$ & $2(4)$ & 0.04 \\
\hline Klebsiella pneumoniae, n (\%) & $1(2)$ & $1(2)$ & 1.00 \\
\hline Staphylococcus haemolyticus, n (\%) & $8(16)$ & $3(6)$ & 0.11 \\
\hline Negativo, n (\%) & $13(26)$ & $32(64)$ & 0.01 \\
\hline
\end{tabular}

Para comparar la diferencia de proporciones entre los grupos se utilizó la prueba de ji al cuadrado.

nacidos a término tienen mayor movilidad. Puede ocurrir una progresión del catéter hasta la aurícula derecha, en algunas ocasiones con perforación secundaria y paso de soluciones hiperosmolares; la fuga de líquido al pericardio ocasiona una compresión cardiaca y taponamiento ${ }^{25}$, que se ha reportado en un $1 \%^{8}$. En este estudio se encontró un porcentaje similar en los recién nacidos pretérmino y a término. Algunos autores reportan el derrame pleural por extravasación del líquido de perfusión en un $1 \%$ de los casos $^{8}$. En nuestra investigación se encontró en un $2 \%$ en ambos grupos de estudio. La rotura del catéter percutáneo en la zona de inserción se reporta en un $9 \%$, yen este estudio se encontró una mayor proporción en los recién nacidos a término que en los pretérmino, debido a que se infunde mayor cantidad de soluciones y se ejerce mayor presión. La oclusión del catéter percutáneo se reporta en un $19 \%^{6}$, y en este estudio se encontró una
Tabla 5. Vena utilizada para insertar el catéter percutáneo en los recién nacidos pretérmino y a término

\begin{tabular}{|c|c|c|c|}
\hline \multirow[t]{2}{*}{ Vena del catéter percutáneo } & \multicolumn{2}{|c|}{ Grupos } & \multirow[t]{2}{*}{ p } \\
\hline & $\begin{array}{c}\text { Pretérmino } \\
n=50\end{array}$ & $\begin{array}{c}\text { Término } \\
n=50\end{array}$ & \\
\hline Axilar derecha, n (\%) & $6(12)$ & $5(10)$ & 0.74 \\
\hline Axilar izquierda, n (\%) & $5(10)$ & $5(10)$ & 1.00 \\
\hline Basílica derecha, n (\%) & $4(8)$ & $0(0)$ & 0.05 \\
\hline Basílica izquierda, n (\%) & $1(2)$ & $0(0)$ & 0.50 \\
\hline Braquial anterior derecha, $\mathrm{n}(\%)$ & $1(2)$ & $9(18)$ & 0.00 \\
\hline Braquial anterior izquierda, n (\%) & $0(0)$ & $1(2)$ & 0.50 \\
\hline Cefálica accesoria derecha, n (\%) & $3(6)$ & $0(0)$ & 0.12 \\
\hline Cefálica accesoria izquierda, n (\%) & $4(8)$ & $1(2)$ & 0.18 \\
\hline Cefálica braquial derecha, n (\%) & $0(0)$ & $1(2)$ & 0.50 \\
\hline Cefálica derecha, n (\%) & $3(6)$ & $3(6)$ & 0.70 \\
\hline Cefálica izquierda, n (\%) & $2(4)$ & $1(2)$ & 0.50 \\
\hline Cefálica mediana derecha, n (\%) & $0(0)$ & $2(4)$ & 0.24 \\
\hline Cefálica mediana izquierda, n (\%) & $0(0)$ & $2(4)$ & 0.24 \\
\hline Mediana basílica derecha, n (\%) & $0(0)$ & $3(6)$ & 0.12 \\
\hline
\end{tabular}

proporción mayor en los pretérmino; puede ser ocasionada por trombos sanguinolentos o por la formación de fibrina, debido a la presencia de sangre en el catéter después de un proceso inadecuado de lavado del catéter o por flujo retrogrado. Además, también puede ser no trombotica, ocasionada por minerales precipitados provenientes de soluciones infundidas o de medicamentos ${ }^{9,10}$. La Infiltración se reporta de un $5 \%^{6}$, y en este estudio se encontró mayor proporción en los pretérmino; esta se debe al desplazamiento del catéter desde la íntima de la vena hacia el tejido subcutáneo.

Una limitación de esta investigación es no haber realizado un seguimiento de las complicaciones en los recién nacidos posterior al retiro del catéter percutáneo, lo cual es útil para identificar complicaciones tardías.

Las ventajas de utilizar el catéter percutáneo son que proporciona un acceso venoso seguro y evita el dolor por múltiples punciones. Es necesario continuar monitorizando la presencia de eventos adversos tempranos y tardíos por la inserción de catéteres percutáneos. Esto ayudará a diseñar nuevas estrategias para colocarlos y corregir los posibles errores, lo cual permitirá que los recién nacidos tengan menores complicaciones y sean integrados a su núcleo familiar 
en forma temprana y sin secuelas. Para la institución, disminuirán los días de hospitalización y los costos.

\section{Conclusiones}

Los resultados del presente estudio demuestran una mayor asociación del catéter percutáneo con las infecciones e infiltraciones en los recién nacidos pretérmino. En los recién nacidos a término se encontraron el retiro accidental y la ruptura del catéter. Es necesario investigar el origen de estas complicaciones para establecer estrategias de prevención.

\section{Conflictos de intereses}

Los autores declaran no tener conflicto de intereses.

\section{Bibliografía}

1. Menon G. Neonatal long lines. Arch Dis Child Fetal Neonatal 2003;88:260-2.

2. Shaw JC. Parenteral nutrition in the management of sick low birth weigth infants. Pediatr Clin North Am. 1973;20:333-58.

3. Neubauer AP. Percutaneous central iv in the neonate: experience with 535 silastic catheters. Acta Paediatr. 1995;84:756-60.

4. Mahieu LM, De Muynck AO, leven MM, et al. Risk factors for centra vascular catheter-associated bloodstream infections among patients in a neonatal intensive care unit. J Hosp Infect. 2001;48:108-6.

5. López Sastre JL, Fernández Colomer B, Coto Cotallo GD, et al. Prospective evaluation of percutaneous central venous catheters in newborn infants. An Esp Pediatr. 2000;53:138-47.

6. Franceschi AT, da Cunha ML. Adverse events related to the use of central venous catheters in hospitalized newborns. Rev Lat Am Enfermagem. 2010;18:196-202.

7. Darling JC, Newell SJ, Mohamdee O, et al. Central venous catheter tip in the right atrium: a risk factor for neonatal cardiac tamponade. J Perinatol 2001;21:461-4.

8. Leipälä JA, Petäjä J, Fellman V. Perforation complications of percutaneous central venous catheters in very low irthweight infants. J Paediatr Child Health. 2001;37:168-71.
9. Smirk C, Soosay Raj T, Smith AL, et al. Neonatal percutaneous central venous lines: fit to burst. Arch Dis Child Fetal Neonatal. 2009;94:298-300.

10. Phillips LD. Catéteres de acesso venoso central. En: Phillips LD. Manual de terapia intravenosa. Porto Alegre (RS): Artmed; 2001. p. 334-72.

11. Ballard JL, Khoury JC, Wedig K, et al. New Ballard Score, expanded to include extremely premature infants. J Pediatrics. 1991;119:417-23.

12. Goldstein B, Giroir B, Randolph A. International pediatric sepsis consensus conference: definitions for sepsis and organ dysfunction in pediatrics. Pediatr Crit Care Med. 2005;6:2-8.

13. World Medical Association. World Medical Association Declaration of Helsinki. Ethical principles for medical research involving human subjects. Nurs Ethics. 2002;9:105-9.

14. Eggimann $\mathrm{P}, \mathrm{Sax} H$, Pittet D. Catheter-related infections. Microbes Infect. 2004;6:1033-42.

15. Yeung CY, Lee HC, Huang FY, et al. Sepsis during total parenteral nutrition: exploration of risk factors and determination of the effectiveness of peripherally inserted central venous catheters. Pediatr Infect Dis J. 1998;17:135-42.

16. Hernández R, Águila E. Estudio prospectivo de las complicaciones infecciosas en RN con catéteres de silicona utilizados para infusión de nutrición parenteral. Anal Esp Pediatr. 1996;45:626-30.

17. Sitges-Serra, Linares J. Catheter sepsis: the clue is the hub. Surgery. 1985;97:355-7.

18. Sitges-Serra A, Puig $P$, Jaurrieta $E$, et al. Catheter sepsis due to Staphylococcus epidermidis during parenteral nutrition. Surg Gynecol Obstet. 1980;151:481-3.

19. Ponnusamy V, Venkatesh V, Curley A, et al. Segmental percutaneous central venous line cultures for diagnosis of catheter-related sepsis. Arch Dis Child Fetal Neonatal. 2012:97:273-8.

20. Nadroo AM, Glass RB, Lin J, et al. Changes in upper extremity position cause migration of peripherally inserted central catheters in neonates. Pediatrics. 2002;110:131-6.

21. Coit AK, Kamitsuka MD. Peripherally inserted central catheter using the saphenous vein: importance of two-view radiographs to determine the tip location. J Perinatol. 2005;25:674-6.

22. Colacchio K, Deng Y, Northrup V, et al. Complications associated with central and non-central venous catheters in a neonatal intensive care unit. J Perinatol. 2012;32:941-6.

23. Jain A, Deshpande $P$, Shah P. Peripherally inserted central catheter tip position and risk of associated complications in neonates. $\mathrm{J}$ Perinatol. 2013:33:307-12

24. Srinivasan HB, Tjin-A-Tam A, Galang $R$, et al. Migration patterns of peripherally inserted central venous catheters at 24 hours postinsertion in neonates. Am J Perinatol. 2013;10:871-4.

25. Chatel-Meijer MP, Roques-Gineste M, Fries F, et al. Cardiac tamponade secondary to umbilical venous catheterization accident in apremature infant Arch Fr Pediatr. 1992;49:373-6. 